1970-02-01

\title{
Asymptotic Evaluation of WKB Matrix Elements. II. Use of Langer's Uniform Asymptotic Wavefunctions
}

Wesley D. Smith

smithw@byui.edu

Russell T. Pack

Follow this and additional works at: https://scholarsarchive.byu.edu/facpub

Part of the Biochemistry Commons, and the Chemistry Commons

\section{Original Publication Citation}

Smith, Wesley D. and Russell T. Pack. "Asymptotic Evaluation of WKB Matrix Elements. II. Use of Langer's Uniform Asymptotic Wavefunctions." The Journal of Chemical Physics 52 (197):

1381-1386.

\section{BYU ScholarsArchive Citation}

Smith, Wesley D. and Pack, Russell T., "Asymptotic Evaluation of WKB Matrix Elements. II. Use of Langer's Uniform Asymptotic Wavefunctions" (1970). Faculty Publications. 798.

https://scholarsarchive.byu.edu/facpub/798

This Peer-Reviewed Article is brought to you for free and open access by BYU ScholarsArchive. It has been accepted for inclusion in Faculty Publications by an authorized administrator of BYU ScholarsArchive. For more information, please contact ellen_amatangelo@byu.edu. 


\title{
Asymptotic Evaluation of WKB Matrix Elements. II. Use of Langer's Uniform Asymptotic Wavefunctions*
}

\author{
Wesley D. Smith and Russell T Pack \\ Department of Chemistry, Brigham Young University, Provo, Ulah 84601
}

(Received 4 August 1969)

\begin{abstract}
An improvement in a previous procedure for the analytic asymptotic evaluation of integrals arising in the quantum-mechanical theory of inelastic molecular collisions is presented. The integrals are evaluated using Langer's uniform asymptotic wavefunctions and the higher-order saddle-point or steepest-descents method. It is found that the Langer functions give no better results than WKB functions, but the higherorder steepest-descents corrections produce a marked improvement. The result is a simple method for evaluating a large class of integrals with an error of $0.1 \%-2.1 \%$.
\end{abstract}

\section{INTRODUCTION}

In order to determine the scattering cross sections in any distorted-wave treatment ${ }^{1}$ of inelastic atomic and molecular collisions, one must evaluate certain integrals (often called $\beta$ integrals ${ }^{2}$ ) of the form

$$
I_{m n}=\int_{0}^{\infty} G_{m}(r) V(r) G_{n}(r) d r,
$$

where $V(r)$ is an off-diagonal matrix element of the perturbing intermolecular potential over internal-state wavefunctions. The scattering wavefunctions $G_{i}(r)$ satisfy

$$
\left(d^{2} G_{i} / d r^{2}\right)+\left(k_{i}{ }^{2}-U_{i}\right) G_{i}=0,
$$

subject to the boundary conditions $G_{i}(0)=0$ and

$$
G_{i}(r) \underset{r \rightarrow \infty}{\longrightarrow} k_{i}^{-1 / 2} \sin \left(k_{i} r-\frac{1}{2} \pi l_{i}+\eta_{i}\right) .
$$

Here $k_{i}=\left(2 \mu E_{i} / \hbar^{2}\right)^{1 / 2}$ is the wavenumber associated with the relative motion of the two particles, $\mu$ their reduced mass, $l_{i}$ their relative orbital angular momentum, and $\eta_{i}$ the phase shift. The effective potential

$$
U_{i}=\left(2 \mu / \hbar^{2}\right) W_{i}(r)+l_{i}\left(l_{i}+1\right) / r^{2}
$$

is the sum of the intermolecular potential and a centrifugal potential.

Two problems are encountered in the evaluation of the $I_{m n}$. First, exact solutions of (2) are not known for most $U_{i}$; one must either find adequate approximate solutions or integrate (2) numerically. Second, the integrand of (1) oscillates rapidly making accurate numerical evaluation difficult and tedious.

In a previous paper ${ }^{3}$ (herein called Paper I), coauthored by one of us, a means was presented by which the $I_{m n}$ were evaluated by asymptotic analytic methods.

\footnotetext{
* This research was supported in part by grants from Research Corporation and from the Brigham Young University Research Fund.

${ }^{1}$ See, e.g., K. Takayanagi, Advan. At. Mol. Phys. 1, 149 (1965).

${ }_{2}^{2}$ N. F. Mott and H. S. W. Massey, The Theory of Atomic Collisions (Oxford University Press, London, 1965), 3rd ed., p. 350.

${ }^{3}$ K. T Pack and J. S. Dahler, J. Chem. Phys. 50, 2397 (1969).
}

WKB wavefunctions were used for the $G_{i}$, and the integration was performed by the lowest-order saddlepoint method along a path on which the integrand did not oscillate. The results were surprisingly good for such a simple method.

In this paper, the same problem is considered, but two changes in procedure are made to improve accuracy. Higher-order terms are included in the saddle-point or steepest-descents method, and Langer's uniform asymptotic wavefunctions ${ }^{4-6}$ are used instead of WKB functions. Langer's functions are everywhere excellent approximations to the solutions of (2); far from the classical turning points they reduce to the WKB solutions, but unlike the WKB functions they remain smooth and continuous near the turning points.

The results obtained are compared with the results of Paper I and with the exact values of $I_{m n}$, using the respulsive exponential potential as an example.

\section{EVALUATION OF THE MATRIX ELEMENTS}

\section{A. The Uniform Asymptotic Wavefunctions}

Let the potentials $U_{i}$ be repulsive in the turningpoint region (see Fig. 1) so that each state has only one turning point. ${ }^{3}$ In this case the appropriate Langer functions are ar $^{4-6}$

$$
G_{i}=\left(\pi / w_{i}{ }^{\prime}\right)^{1 / 2} \mathrm{Ai}\left(-w_{i}\right),
$$

where $\mathrm{Ai}$ is the Airy function, $w_{i}$ is given by

$$
w_{i}(r)=\left(\frac{3}{2} S_{i}\right)^{2 / 3},
$$

and the $S_{i}$ are the action integrals ${ }^{7}$

$$
S_{i}=\int_{r_{i}}^{r} p_{i} d r
$$

\footnotetext{
${ }^{4}$ R. E. Langer, Phys. Rev. 51, 669 (1937).

${ }^{5}$ For good discussions of uniform asymptotic functions and of phase-integral (WKB) methods, see R. B. Dingle, Appl. Sci. Res. B5, 345 (1965); and J. Heading, An Introduction to PlaseIntegral Methods (Methuen and Co., Ltd,, London, 1962), respectively.

${ }^{\circ}$ A. Erdelyi, Asymptotic Expansions (Dover Publications, Inc., New York, 1956), p. 98.

7 This notation for the $S_{i}$ differs by a phase factor from the
} 381 


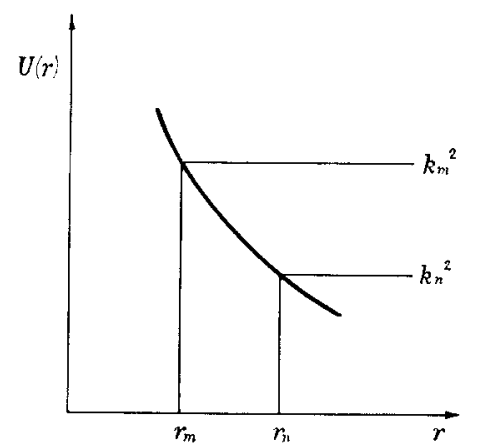

FIG. 1. The relationship of the turning points $r_{m}$ and $r_{n}$ to the wavenumbers $k_{m}$ and $k_{n}$ and to the effective potential energy $U(R)$ in the case of $U_{m}=U_{n}=U$.

with $p_{i}=\left(k_{i}{ }^{2}-U_{i}\right)^{1 / 2}$. The classical turning point $r_{i}$ is defined by $p_{i}\left(r_{i}\right)=0$. The Airy function is an entire function of $w_{i}$ and is real for real $w_{i}$. For real $r>r_{i}, w_{i}$ is real and positive; for real $r<r_{i}, w_{i}$ is real and negative. The function $w^{\prime}=d w_{i} / d r$ is never zero. Therefore, $G_{i}$ is an entire function of $r$ and is real for real $r$.

The Airy functions can be written in terms of Bessel (Hankel) functions in several ways. For example, one convenient form is ${ }^{8}$

$$
G_{i}=\left(\pi S_{i} / 8 p_{i}\right)^{1 / 2}\left[e^{i \pi / 6} H_{1 / 3}{ }^{(1)}\left(S_{i}\right)+e^{-i \pi / 6} H_{1 / 3}{ }^{(2)}\left(S_{i}\right)\right] .
$$

The $H_{1 / 3}{ }^{(1)}$ are analytic functions of $S_{i}$ on $-\pi \leq \arg S_{i}<$ $\pi$. Their complex conjugates have the property that

$$
\left[H_{1 / 3}{ }^{(1)}(z)\right]^{*}=H_{1 / 3}{ }^{(2)}\left(z^{*}\right) .
$$

Hence, for real $r>r_{i}$, which implies that both $S_{i}$ and $p_{i}$ are real, one has

$$
G_{i}=\operatorname{Re}\left(\pi S_{i} / 2 p_{i}\right)^{1 / 2} e^{i \pi / 6} H_{1 / 3}{ }^{(1)}\left(S_{i}\right) .
$$

This equation also holds for real $r<r_{i}$. To see this, consider the behavior of $p_{i}$ and $S_{i}$ starting from $r>r_{i}$ and moving left. Near the turning point, let $z_{i}=r-r_{i}$, and expand $p_{i}$ and $S_{i}$ for small $z_{i}$. The results are

$$
p_{2} \approx\left[-U_{i}^{\prime}\left(r_{i}\right)\right]^{1 / 2 z_{i}{ }^{1 / 2}}
$$

and

$$
S_{i} \approx \frac{2}{3}\left[-U_{i}{ }^{\prime}\left(r_{i}\right)\right]^{1 / 2 z_{i}{ }^{3 / 2}}
$$

thus, as $r$ passes $r_{i}$ in the upper half-plane, $\arg p_{i}$ changes from 0 to $\pi / 2$ and $\arg S_{i}$ changes from 0 to $3 \pi / 2$. This suggests defining the quantities

$\operatorname{and}^{7}$

$$
q_{i}=e^{-i \pi / 2} p_{i}=\left(U_{i}-k_{i}^{2}\right)^{1 / 2}
$$

$$
S_{i}^{<}=\exp \left(-\frac{3 i \pi}{2}\right) S_{i}=\int_{r}^{r_{i}} q_{i} d r
$$

8 Handbook of Mathematical Functions, M. Abramowitz and I. A. Stegun, Eds. (Dover Publications, Inc., New York, 1965), p. 447.
Thus defined, both $q_{i}$ and $S_{i}$ are real and positive for real $r<r_{i}$. Then (8) becomes

$$
\begin{aligned}
& G_{i}=\operatorname{Re}\left(\pi S_{i}<e^{i \pi} / 2 q_{i}\right)^{1 / 2} e^{i \pi / 6} H_{1 / 3}^{(1)}\left(e^{3 i \pi / 2} S_{i}^{<}\right), \\
& =\left(\pi S_{i}<e^{i \pi / 8} q_{i}\right)^{1 / 2} e^{i \pi / 6} H_{1 / 3}^{(1)}\left(e^{3 i \pi / 2} S_{i}<\right) \\
& +\left(\pi S_{i}^{<} e^{-i \pi} / 8 q_{i}\right)^{1 / 2} e^{-i \pi / 6} H_{1 / 3}^{(2)}\left(e^{-3 i \pi / 2} S_{i}<\right), \\
& =\left(\pi S_{i} / 8 p_{i}\right)^{1 / 2}\left[e^{i \pi / 6} H_{1 / 3}{ }^{(1)}\left(S_{i}\right)-e^{-i \pi / 6} H_{1 / 3}{ }^{(2)}\left(e^{-3 \pi i} S_{i}\right)\right] .
\end{aligned}
$$

Using the analytic continuation formulas ${ }^{9}$ for $H_{1 / 3}{ }^{(2)}$ in (13), one recovers (6) as asserted.

We can also write (3) in terms of Bessel functions another convenient way. By letting $S_{i}=e^{-i \pi} S_{i}$ in (6) and by employing the analytic continuation formulas ${ }^{9}$ one obtains

$$
G_{i}=\left(\pi S_{i} / 8 p_{i}\right)^{1 / 2} e^{i \pi / 6} H_{1 / 3}^{(1)}\left(S_{i}\right) .
$$

In this expression, $\arg S_{i}$ stays in the domain on which the Hankel functions are analytic for all $r$ of interest.

\section{B. The Matrix Elements}

Consider a typical molecular collision problem in which $U_{m}$ nearly equals $U_{n}$ and in which $k_{m}>k_{n}$, so that $r_{m}<r_{n}$ (see Fig. 1). In this case it was shown in Paper I that the WKB integrand of (1) could be put into a form which had a saddle point at $R<r_{m}$. Since the Langer functions reduce to the WKB functions except near the turning points, the Langer integrand of (1) is expected to behave similarly. Hence, we split the integral into two parts:

where

$$
I_{m n}=I_{1}+I_{2},
$$

$$
I_{1}=\int_{0}^{R} G_{m}(r) V(r) G_{n}(r) d r
$$

and

$$
I_{2}=\int_{R}^{\infty} G_{m}(r) V(r) G_{n}(r) d r
$$

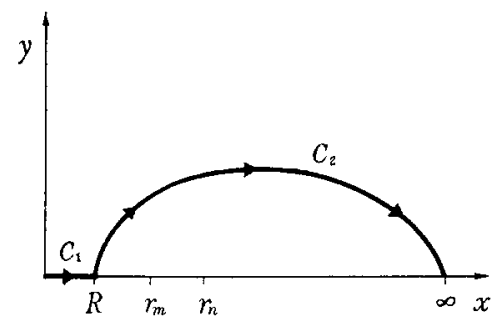

Fig. 2. The contour of integration for the case $U_{n}=U_{m}$. $R$ is the saddle point; $C_{2}$ is the path of steepest descents. It starts parallel to the imaginary axis but curves to the right faster than the lowest order path of steepest descents used in Paper I.

${ }^{9}$ Reference 8, p. 361. 
By using (14) for both $G_{m}$ and $G_{n}$ in (16), we can write $I_{1}$ in the form

$I_{1}=\left(\frac{1}{8} \pi\right) e^{i \pi / 3} \int_{0}^{R} V(r)\left(\frac{S_{m} S_{n}}{p_{m} p_{n}}\right)^{1 / 2} H_{1 / 3}{ }^{(1)}\left(\S_{m}\right) H_{1 / 3}{ }^{(1)}\left(S_{n}\right) d r$.

This integrand dies exponentially on this interval making $I_{1}$ very small and readily estimated using either numerical or analytic methods.

Since $G_{n}$ and the range of integration are both real, we can substitute (8) for $G_{m}$ in (17) and then (14) for $G_{n}$ to obtain ${ }^{10}$

$$
\begin{aligned}
I_{2}=\operatorname{Re}\left(\frac{1}{4} \pi\right) e^{i \pi / 3} \int_{R}^{\infty} & V(r)\left(\frac{S_{m} S_{n}}{p_{m} p_{n}}\right)^{1 / 2} \\
& \times H_{1 / 3}{ }^{(1)}\left(S_{m}\right) H_{1 / 3}{ }^{(1)}\left(S_{n}\right) d r .
\end{aligned}
$$

The evaluation of this integral is greatly facilitated by the fact that the integrand has a saddle point at $R$ on the real axis. [Substitution of either (3) or (6) into (1) yields an integrand which does not.] In addition, the integrand is an analytic function, so that we are free (by Cauchy's theorem) to deform the contour of integration to follow $C_{2}$, the path of steepest descents, as shown in Fig. 2. On such a path the integrand no longer oscillates, and the difficulties inherent in (1) are avoided.

At this point probably the most accurate method of evaluating $I_{m n}$ would be by numerical contour integration of $I_{1}$ and $I_{2}$ along $C_{1}$ and $C_{2}$, respectively. However, it is usually possible to continue analytically and obtain a value for $I_{m n}$ which has better accuracy than is either needed or justified by the distorted-wave approximation that gave rise to the integrals.

Let us first consider $I_{1}$. Since $I_{1}$ is expected to be small (it was neglected in Paper I), and since the saddle point $R$ usually lies far to the left of $r_{m}, I_{1}$ can be approximated with sufficient accuracy by employing the first terms of the Hankel functions' asymptotic expansions ${ }^{11}-$ the WKB functions. Changing to the real quantities $q_{i}$ and $S_{i}<$, one then finds that

$I_{1} \sim \frac{1}{4} \int_{0}^{R} V(r)\left(q_{m} q_{n}\right)^{-1 / 2} \exp \left(-S_{m}<-S_{n}<\right) d r$.

An asymptotic representation of $I_{1}$ can be obtained by the method of integration by parts. ${ }^{12,13}$ We write

$$
\begin{aligned}
I_{1} \sim \frac{1}{4} \int_{0}^{R} d r V(r)\left(q_{m} q_{n}\right)^{-1 / 2}\left(q_{m}+q_{n}\right)^{-1} & \\
& \times\left(\frac{d}{d r}\right) \exp \left(-S_{m}<-S_{n}<\right) .
\end{aligned}
$$

${ }^{10} \mathrm{It}$ is assumed that $V(r)$ is real.

11 Reference 8, p. 364 .

12 Reference 6, p. 26.

${ }^{13} \mathrm{E}$. T. Copson, Asymptotic Expansions (Cambridge University Press, Cambridge, England, 1967), p. 13.
Integrating by parts and noting that $\exp \left(-S_{i}<\right) \rightarrow 0$ as $r \rightarrow 0$ for most potentials, we find that

$$
\begin{aligned}
& I_{1} \sim \frac{1}{4} V(R)\left[q_{m}(R) q_{n}(R)\right]^{-1 / 2} \\
& \quad \times\left[q_{m}(R)+q_{n}(R)\right]^{-1} \exp \left[-S_{m}<(R)-S_{n}^{<}<(R)\right] \\
& \quad-\frac{1}{4} \int_{0}^{R}\left(\frac{d}{d r}\left[V(r)\left(q_{m} q_{n}\right)^{-1 / 2}\left(q_{m}+q_{n}\right)^{-1}\right]\right) \\
& \quad \times \exp \left(-S_{m}<-S_{n}<\right) d r .
\end{aligned}
$$

The integrand of the second term can be rewritten in a form involving the derivative of the exponentials and again integrated by parts. Repetition of this process yields an asymptotic series for $I_{1}$, but for our purposes the first term is sufficient. Thus, we have

$$
\begin{aligned}
& I_{1} \sim \frac{1}{4} V(R)\left[q_{m}(R) q_{n}(R)\right]^{-1 / 2} \\
& \quad \times\left[q_{m}(R)+q_{n}(R)\right]^{-1} \exp \left[-S_{m}<(R)-S_{n}<(R)\right] .
\end{aligned}
$$

Next let us evaluate $I_{2}$ by the method of steepest descents. If the path of integration $C_{2}$ stays sufficiently far from the turning points, the Hankel functions in (19) can be replaced by their asymptotic expansions. ${ }^{11}$ In terms of $q_{i}$ and $S_{i}^{<},(19)$ can then be written

$$
\begin{aligned}
I_{2} \sim-\operatorname{Re}_{2} \frac{1}{2} i \int_{C_{2}} V(z) & \left(q_{m} q_{n}\right)^{-1 / 2} \\
& \times \exp \left(S_{m}^{<}-S_{n}^{<}\right) D_{m} C_{n} d z,
\end{aligned}
$$

where $r=z$ has become a complex variable. This equation is now identical to the WKB approximation of Paper I except for the correction terms $D_{m}$ and $C_{n}$ defined by the asymptotic series ${ }^{14}$

$$
\begin{aligned}
D_{m}=1+0.069444\left(S_{m}<\right)^{-1} & +0.037134\left(S_{m}<\right)^{-2} \\
& +0.037993\left(S_{m}<\right)^{-3}+\cdots
\end{aligned}
$$

and

$$
\begin{aligned}
C_{n}=1-0.069444\left(S_{n}<\right)^{-1} & +0.037134\left(S_{n}<\right)^{-2} \\
& -0.037993\left(S_{n}<\right)^{-3}+\cdots .
\end{aligned}
$$

To perform the steepest-descents evaluation, we write (22) in the form

$$
I_{2}=-\operatorname{Re} \frac{1}{2} i \int_{C_{2}} g(z) \exp [h(z)] d z
$$

${ }^{14} H_{1 / 3}{ }^{(1)}\left(S_{m}\right)$ also contains a dying exponential term. But if $R$ is enough smaller than $r_{m}$ that the use of the asymptotic formulas is valid, the dying term is negligibly smaller than the terms kept. For a discussion of this point, see G. N. Watson, $A$ Treatise on the Theory of Bessel Functions (The Macmillan Company, New York, 1944), 2nd ed., p. 201. It should be noted that neglect of this dying term also implies that $I_{1}$ is negligible because the integrand of $I_{1}$ [see Eq. (20)] is a dying exponential smaller than the one ignored in $I_{2}$. However, we keep $I_{1}$ as an index of the accuracy of this approximation. 
where

$$
h(z)=S_{m}<-S_{n}<+\ln V(z)-\frac{1}{2} \ln q_{m} q_{n},
$$

and

$$
g(z)=D_{m} C_{n} .
$$

Now in the usual higher-order steepest-descents method $g(z)$ and its derivatives would be involved in the determination of the path of steepest descents. However, like many asymptotic series, ${ }^{15}$ those in $g(z)$ are not usually differentiable. Hence, the best estimate possible for the effect of $g(z)$ is that of the lowest-order steepest descents or saddle-point method,

$$
I_{2}=-\operatorname{Re} \frac{1}{2} i g(R) \int_{C_{2}} \exp [h(z)] d z .
$$

However, we do want to find higher-order terms due to $h(z)$. Following Copson, ${ }^{16}$ we let

$$
h(z)=h(R)-t^{2} .
$$

The saddle point $R$ is the point at which $h^{\prime}(R)=0$. The path of steepest descent is that path $C_{2}$ on which $t^{2}$ is real and positive. Thus, (28) becomes

$I_{2}=-\operatorname{Re}_{2}^{\frac{1}{2}} i g(R) \exp [h(R)] \int_{0}^{\infty} \exp \left(-t^{2}\right) \frac{d z}{d t} d t$.

Expanding $d z / d t$ in an asymptotic power series,

$$
\frac{d z}{d t}=\sum_{n=0}^{N} A_{n} t^{n}
$$

one obtains

$$
\begin{aligned}
I_{2} & =-\operatorname{Re} \frac{1}{2} i g(R) \exp [h(R)] \sum_{n=0}^{N} A_{n} \int_{0}^{\infty} \exp \left(-t^{2}\right) t^{n} d t \\
& =-\operatorname{Re}_{4} \frac{1}{i g}(R) \exp [h(R)] \sum_{n=0}^{N} A_{n} \Gamma[(n+1) / 2]
\end{aligned}
$$

The coefficients $A_{n}$ are given by the residues ${ }^{16}$

$$
A_{n}=(2 \pi i)^{-1} \oint[h(R)-h(z)]^{-(n+1) / 2} d z,
$$

in which the path of integration is a small closed contour around $R$. The $A_{n}$ are readily evaluated and expressed in the form

$$
A_{n}=\exp [i \pi(n+1) / 2]\left[2 / h^{(2)}(R)\right]^{(n+1) / 2} Q_{n},
$$

where $h^{(n)}(R)$ are the derivatives of $h(z)$ evaluated at

\footnotetext{
${ }^{15}$ Reference 6, pp. 14-17; N. G. De Bruijn, A symplotic Methods in Analysis (North-Holland Publ. Co., Amsterdam, 1958), p. 17.

${ }^{16}$ Reference 13, p. 65.
}

$R$. The first few $\mathfrak{Q}_{n}$ are

$\mathfrak{Q}_{0}=1$,

$\alpha_{1}=-d_{1}$,

$a_{2}=-\frac{3}{2} d_{2}+(15 / 8) d_{1}^{2}$,

$a_{3}=-2 d_{3}+6 d_{1} d_{2}-4 d_{1}^{3}$,

$\mathfrak{Q}_{4}=-\frac{5}{2} d_{4}+\frac{35}{4} d_{3} d_{1}+\frac{35}{8} d_{2}^{2}-\frac{315}{16} d_{1}^{2} d_{2}+\frac{1155}{128} d_{1}^{4}$,

$\mathfrak{Q}_{5}=-3 d_{5}+12\left(d_{4} d_{1}+d_{3} d_{2}\right)-30\left(d_{1}^{2} d_{3}+d_{1} d_{2}^{2}\right)$

$+60 d_{1}^{3} d_{2}-21 d_{1}^{5}$

$\mathfrak{Q}_{6}=-\frac{7}{2} d_{6}+\frac{63}{4}\left(d_{1} d_{5}+d_{4} d_{2}\right)+\frac{63}{8} d_{3}{ }^{2}-\left(\frac{693}{16}\right) d_{1}^{2} d_{4}$

$-\frac{693}{8} d_{1} d_{2} d_{3}-\frac{231}{16} d_{2}^{3}+\frac{3003}{32} d_{3} d_{1}^{3}+\frac{9009}{64} d_{2}^{2} d_{1}^{2}$

$-\frac{45045}{256} d d_{1}^{4} d_{2}+\frac{51051}{1024} d_{1}^{6}$

where the $d_{n}$ are given by the ratios

$$
d_{n}=2 h^{(n+2)}(R)\left[(n+2) ! h^{(2)}(R)\right]^{-1} .
$$

Now the $a_{n}$ are real and $h^{(2)}(R)$ is positive; thus, $I_{2}$ becomes

$$
\begin{aligned}
I_{2}=\frac{1}{4} g(R) \exp [h(R)] \sum_{n=0}^{N} & \left(\frac{2}{h^{(2)}(R)}\right)^{(n+1) / 2} \\
& \times \Gamma\left(\frac{(n+1)}{2}\right) a_{n} \operatorname{Re}\left(e^{i n \pi / 2}\right) .
\end{aligned}
$$

Since only terms with $n$ even will make any contribution, we let $n=2 k$ and write

$I_{2}=\frac{1}{4} g(R) \exp [h(R)]$

$$
\times \sum_{k=0}^{K}(-1)^{k}\left(\frac{2}{h^{(2)}(R)}\right)^{k+1 / 2} \Gamma\left(k+\frac{1}{2}\right) \mathfrak{a}_{2 k} .
$$

The first term in the sum is the result of Paper I. If it is factored out, we can write

$$
I_{2}=\mathfrak{g} g(R) T(R)
$$

where

$$
\mathfrak{I}=\frac{1}{2} \exp [h(R)]\left[\pi / 2 h^{(2)}(R)\right]^{1 / 2}
$$

is the approximation for $I_{m n}$ obtained in Paper I, and,

$$
T(R)=\sum_{k=0}^{K}(-1)^{k}\left(\frac{2}{h^{(2)}(R)}\right)^{k} \pi^{-1 / 2} \Gamma\left(k+\frac{1}{2}\right) \mathfrak{Q}_{2 k}
$$

is a correction due to the higher-order steepest-descent method. The series $T(R)$ converges for a few terms and then begins to diverge - a behavior typical of asymptotic series. The maximum accuracy possible with such series is achieved by truncating them at the smallest term, and the error is of the same order of magnitude as that smallest term. ${ }^{17}$ The other asymptotic series in (39),

${ }^{17}$ Reference 6, p. 11; Ref. 13, p. 6. 
$g(R)$, which represents the difference between the Langer functions and the WKB functions, is a correction for the divergences of the WKB functions.

We have thus obtained formulas (21) and (39) as an asymptotic estimate of $I_{m n}=I_{1}+I_{2}$. The determintion of the $S_{i}$ (discussed in Paper I) completes the evaluation of the matrix element.

\section{EXAMPLE. THE REPULSIVE EXPONENTIAL POTENTIAL}

Consider again the example used in Paper I. Let $l_{m}=l_{n}=0, W_{m}=W_{n}=C \exp (-r / a)$, and $V=\left(2 \mu / \hbar^{2}\right) W_{i}$. If $r$ is measured in units of $a$ (about $0.2 \AA$ ) and $E$ in units of $\hbar^{2} / 2 \mu a^{2}$ (typically about $40^{\circ} \mathrm{K}$ ), then one has $E_{i}=k_{i}{ }^{2}$ and $U=D e^{-r}$ with $D \approx 4 \times 10^{8}$. In these units typical vibrational and rotational constants are $\theta_{v}=$ $2000^{\circ} \mathrm{K}=50$ and $\theta_{r}=2^{\circ} \mathrm{K}=0.05$.

Jackson and Mott ${ }^{18}$ have solved both (2) and (1) exactly for this potential. We now compare our asymptotic results with their exact formula in the limits of large (vibrational) energy exchange and small (resonance) energy exchange. The results for rotational transitions lie between those of these two limits but are not discussed here because they do not reduce to simple formulas.

\section{A. Vibrational Transitions}

If vibrational energy is exchanged, we have $k_{m}{ }^{2}-$ $k_{n}{ }^{2} \geq 50$, and for energies even slightly above threshold, the exact result ${ }^{18}$ reduces to $^{3}$

$$
I_{m n}(\text { exact })=\pi\left(k_{m}^{2}-k_{n}^{2}\right) \exp \left[-\left(k_{m}-k_{n}\right) \pi\right] \text {. }
$$

As in Paper I, the saddle point $R$ is determined to a good approximation by

$$
U(R)=\left(k_{m}^{2}-k_{n}^{2}\right)^{2},
$$

and the WKB lowest-order saddle-point result of Paper $I$ is

$$
\mathfrak{J}=e(2 \pi)^{-1 / 2} I_{m n}(\text { exact }) ;
$$

hence, $I_{m n}$ (asymptotic), as obtained in the previous

Table I. Correction terms for vibrational transitions with $k_{m}^{2}-k_{n}{ }^{2}=50$.

\begin{tabular}{crrl}
\hline \hline $\begin{array}{c}E_{n} \\
\left({ }^{\circ} \mathrm{K}\right)\end{array}$ & $k_{n}{ }^{2}$ & $k_{m}{ }^{2}$ & $g(R)$ \\
\hline 40 & 1 & 51 & 1.0002 \\
160 & 4 & 54 & 1.0002 \\
280 & 7 & 57 & 1.0001 \\
360 & 9 & 59 & 1.0001 \\
640 & 16 & 66 & 1.0001 \\
1440 & 36 & 86 & 1.0001 \\
4000 & 100 & 150 & 1.0001 \\
\hline \hline
\end{tabular}

${ }^{18} \mathrm{~J}$. M. Jackson and N. F. Mott, Proc. Roy. Soc. (London) A137, 703 (1932).
TABI.E II. Correction terms for resonance transitions with $k_{m}^{2}=k_{n}^{2}=k^{2}$

\begin{tabular}{rrrr}
\hline \hline $\begin{array}{c}E_{i} \\
\left({ }^{0} \mathrm{~K}\right)\end{array}$ & $k^{2}$ & $g(R)$ & $I_{\mathrm{I}}$ \\
\hline 40 & 1 & 0.9738 & 0.1060 \\
160 & 4 & 1.0968 & 0.0449 \\
280 & 7 & 1.0508 & 0.0272 \\
360 & 9 & 1.0402 & 0.0190 \\
640 & 16 & 1.0366 & 0.0081 \\
1440 & 36 & 1.0130 & 0.0013 \\
4000 & 100 & 1.0041 & 0.0001 \\
\hline
\end{tabular}

section of this paper, becomes

$I_{m n}($ asymptotic $)=I_{1}+g(R) T(R) e(2 \pi)^{-1 / 2} I_{m n}$ (exact).

Using (43) one finds that for $n \geq 2$,

$$
h^{(n)}(R)=2^{-n}
$$

approximately. Upon evaluating the $A_{2 k}$, we then obtain

$$
\begin{aligned}
T(R) & =(1-1 / 12+0.0034722+0.0026813+\cdots), \\
& =(0.92282),
\end{aligned}
$$

where we have truncated at the smallest term $(K=3)$.

The correction term $g(R)$ depends on the values of $k_{n}$ and $k_{m}$ and does not reduce to a simple expression. Its values can be calculated using (23), (24) and the formula ${ }^{3}$

$S_{i}^{<}=2\left[U(R)-k_{i}{ }^{2}\right]^{1 / 2}-2 k_{i} \tan ^{-1}\left\{\left[U(R)-k_{i}{ }^{2}\right]^{1 / 2} k_{i}{ }^{-1}\right\}$.

Typical values, which are always slightly greater than one, are tabulated in Table $\mathbf{I}$.

The integral $I_{1}$ also depends on $k_{n}$ and $k_{m}$ and does not reduce to a constant. However, in this case $U_{i}(R)$ is very large, and one can readily see from (21) and (48) that $I_{1} \sim e^{-100}$ is completely negligible for vibrational transitions.

Finally, substituting (47) for $T(R)$ in (45) and 1.0001 for $g(R)$, we have

$$
I_{m n}(\text { asymptotic })=1.0008 I_{m n}(\text { exact }) \text {. }
$$

However, if we neglect the Langer corrections to the WKB approximation by setting $g(R)=1$, the WKB, corrected by the higher-order steepest-descents terms only, is just as accurate:

$$
I_{m n}(\mathrm{WKB})=\mathfrak{g} T(R)=1.0007 I_{m n}(\text { exact }) .
$$

\section{B. Resonant Transfer of Internal Energy}

For resonant transition, $k_{m}^{2}=k_{n}^{2}=k^{2}$, and the exact result reduces to ${ }^{3}$

$$
I_{m n}(\operatorname{exact})=k \text {. }
$$


In this case $R$ is defined by $U(R)=2 k^{2}$ and the result of Paper I is

$$
\mathfrak{S}=\left(\frac{1}{2} \pi\right)^{1 / 2} I_{m n}(\text { exact }) ;
$$

thus, the result of this paper is

$$
I_{m n}(\text { asymptotic })=I_{1}+g(R) T(R)\left(\frac{1}{2} \pi\right)^{1 / 2} I_{m n}(\text { exact }) .
$$

Here the $h^{(n)}(R)$, for $n=2$ through 6 , are $1,3,13,75$, and 541, respectively, so that from (41),

$$
\begin{aligned}
T(R) & =\left(1-\frac{1}{4}+0.03125+\cdots\right) \\
& =0.78125,
\end{aligned}
$$

where we have again truncated the sum at the smallest term $(K=2)$. Ignoring $g(R)$ and $I_{1}$, we find

$$
I_{m n}(\mathrm{WKB})=\mathfrak{g} T(R)=0.9792 I_{m n}(\mathrm{exact}) .
$$

The corrections $I_{1}$ and $g(R)$ are obtained by noting that the action integrals in this case reduce to

$$
S_{m}<=S_{n}<=(2-\pi / 2) k=0.4292 k,
$$

so that (21) becomes

$$
I_{1} \sim \frac{1}{4} \exp (-0.8584 k) .
$$

Values of $g(R)$ and $I_{1}$ calculated for a range of $k$ values are listed in Table II. It is clear that in

$$
I_{m n} \text { (asymptotic) }=I_{1}+g(R)(0.9792) I_{m n}(\text { exact })
$$

they make significant changes, but that those changes do not constitute any systematic improvement.

\section{DISCUSSION}

From the results on the example problem we can make a number of observations and conclusions which should apply to any potential which has a shape similar to the repulsive exponential. First, the corrections $I_{1}$, due to the exponentially dying part of the integrand, and $g(R)$, due to the use of the Langer functions, do not improve the results; they serve only as indices of the validity of the asymptotic approximations used..$^{14}$ Equally good or better results are obtained much more simply using WKB functions (including higher-order steepest-descents terms). Second, the accuracy obtained with the WKB functions, $0.07 \%-2.08 \%$, indicates that asymptotic methods and asymptotic wavefunctions are capable of giving very nearly exact results. [It is possible that the $0.07 \%$ is somewhat fortuitous. Both (43) and (44) are approximate, and numerical calculations indicate that these approximations may change the asymptotic result by almost $1 \%$ ]. In either case, the error is considerably less than the error inherent in the distorted-wave approximation which is implied when the integrals (1) are used to calculate cross sections. These asymptotic methods are easily applied to different problems; hence, laborious numerical integration of (2) and (1) should usually be unnecessary.

Finally, let us comment on some earlier work using Langer and WKB functions. Using methods similar to those presented here and in Paper I, Shin ${ }^{19}$ has evaluated matrix elements equivalent to these for the repulsive exponential potential. The error in his results was a few tenths of a percent at higher energies but became much larger at lower energies. However, as Shin noted, and as (50) also shows, this error was due to approximations made in evaluating the action integrals $S_{i}$ and was not due to the WKB approximation.

Langer's wavefunctions have been used by Hartmann and Slawsky ${ }^{20}$ in the numerical integration of matrix elements equivalent to $I_{m n}$ using the SSH potential,

$$
W=C \exp (-\boldsymbol{r} / a)-\boldsymbol{\epsilon} .
$$

Their results differ from the exact results by $0.4 \%$ at high energies but as much as $22.3 \%$ at low energies. Since the SSH potential differs from the repulsive exponential only by a constant, one has the same equation (2) to solve for the $G_{i}$ except that $E_{i}$ is replaced by $E_{i}+\epsilon$. Hence, our analysis using this potential gives the same formulas as those for the repulsive exponential potential except with a shifted $k_{i}$. Furthermore, (42) is valid for the entire energy range they considered. Hence, our (49) holds, and it is clear that the large error in Hartmann and Slawsky's result at low energies is an error in their numerical integration rather than in the Langer functions. Shin ${ }^{19}$ has attempted unsuccessfully to match Hartmann and Slawsky's results for the SSH potential using WKB functions. From (50) we see that the discrepancy is not a consequence of the WKB approximation but is probably due to Shin's use of a different range parameter, $a$.

\section{ACKNOWLEDGMENT}

We thank Professor John S. Dahler for his suggestions and encouragement on this problem.

${ }^{19}$ H. K. Shin, J. Chem. Phys. 48, 3644 (1968).

${ }^{20}$ B. Hartmann and Z. I. Slawsky, J. Chem. Phys. 47, 2491 $(1967)$. 